\title{
Editorial
}

\section{Optical Diagnosis of Diminutive Colorectal Polyps: Can Any Old Dog Learn This New Trick?}

\author{
Inês Pita ${ }^{a}$ Pedro Pimentel-Nunes ${ }^{a-c}$ \\ a Department of Gastroenterology, Portuguese Oncology Institute, Porto, Portugal; ${ }^{b}$ Department of Surgery and \\ Physiology, Porto Faculty of Medicine, Porto, Portugal; ${ }^{C}$ CINTESIS, Porto Faculty of Medicine, Porto, Portugal
}

Keywords

Endoscopy · Optical diagnosis · Narrow-band imaging · Virtual chromoendoscopy

\section{Diagnóstico endoscópico de pólipos colorrectais diminutos: disseminável a qualquer endoscopista?}

\section{Palavras Chave}

Endoscopia - Diagnóstico óptico · Narrow-band imaging · Cromoendoscopia virtual

In this issue of GE - Portuguese Journal of Gastroenterology, Castela et al. [1] report on the diagnostic accuracy of the real-time optical diagnosis of colorectal polyps using the NBI International Colorectal Endoscopic (NICE) and the Workgroup on serrAted polypS and Polyposis (WASP) classification.

One of the practical applications of real-time optical diagnosis is implementing the "diagnose-and-leave" and the "resect-and-discard" strategies during screening and surveillance colonoscopies. In the first, diminutive $(\leq 5$ $\mathrm{mm}$ ) rectosigmoid hyperplastic polyps (HPs) are left in situ, as they are considered to have no malignant potential. In the second, diminutive neoplastic colorectal polyps are resected but not sent for histopathological analysis, as they rarely harbor advanced histology (high-grade dysplasia or a villous component), and post-polypectomy surveillance intervals are assigned based on optical diagnosis. This can reduce the cost, risk, and workload associated with colonoscopy. The American Society for Gastrointestinal Endoscopy has defined the minimal thresholds necessary to safely incorporate them in clinical practice: a negative predictive value (NPV) of $\geq 90 \%$ for neoplastic polyps in the "diagnose-and-leave" strategy and a $\geq 90 \%$ agreement between optical-based and pathology-based surveillance intervals for the "resect-and-discard" strategy [2].

In practice, these policies require accurate optical differentiation between nonneoplastic (HPs) and neoplastic lesions (adenomas [ADs] and sessile serrated lesions, SSLs). NICE classification was validated for the optical distinction of HPs and ADs; however, it does not include SSLs. The WASP was developed and validated in 2015 to differentiate SSLs from HPs and ADs [3].

\section{KARGER}

E-Mail karger@karger.com www.karger.com/pjg (c) 2019 Sociedade Portuguesa de Gastrenterologia Published by S. Karger AG, Basel

Karcer

Open access

This article is licensed under the Creative Commons AttributionNonCommercial-NoDerivatives 4.0 International License (CC BYNC-ND) (http://www.karger.com/Services/OpenAccessLicense). Usage and distribution for commercial purposes as well as any distribution of modified material requires written permission.
Inês Pita, Serviço de Gastrenterologia

Instituto Português de Oncologia de Francisco Gentil

R. Dr. António Bernardino de Almeida 62

PT-4200-162 Porto (Portugal)

E-Mail neispita@ hotmail.com 
Castela et al. [1] evaluated the accuracy of optical diagnosis of diminutive polyps by thirteen endoscopists (trainees and attendings) using both the NICE and WASP classifications in two timepoints 1 year apart. There was an initial 20-min audiovisual training session on these classifications prior to the study's beginning but no subsequent formal training or feedback. Polyps were classified based on optical characteristics as ADs, SSLs, or HPs. A total of 290 polyps were analyzed; 192 in the first study period and 98 in the second. The overall diagnostic accuracy was $75 \%$ in the first period, with a nonstatistically significant increase to $82 \%$ in the second time point; highconfidence diagnoses fared a bit better (70 and $91 \%$, respectively). Accuracy was higher for SSLs ( $>90 \%$ in both study periods and regardless of high-confidence predictions) and remained between $78-86 \%$ for ADs and HPs. The NPV for ADs failed to reach the Preservation and Incorporation of Valuable Endoscopic Innovations (PIVI) threshold in both periods, even for high-confidence diagnoses. There was a nonsignificant NPV improvement from $61 \%$ (95\% CI $46-74)$ to $83 \%$ (95\% CI 65-94) between the study periods for high-confidence predictions.

This study has several strengths. It is one of the few publications on the optical diagnosis of diminutive polyps that includes both the NICE and WASP classifications. While the "diagnose-and-leave" and "resect-anddiscard" strategies were initially focused on the distinction between hyperplastic and adenomatous polyps, the importance of serrated lesions as colorectal cancer precursors is widely recognized, and they can be misdiagnosed as hyperplastic both endoscopically and histopathologically. It also reflects the real-world use of the NICE and WASP during routine consecutive colonoscopies by endoscopists with no previous NBI experience. The study's endoscopists could not achieve the PIVI threshold for this strategy; however, the results are likely influenced by the small number of polyps analyzed, as evidenced by the relatively wide confidence intervals.

As the study's main strength is the inclusion of the WASP classification, it might have been more appropriate to report the NPV for neoplastic lesions (both ADs and SSLs, instead of ADs only), as only HPs are supposed to be left in situ in the "do-not-resect" strategy. The definition of the study's participants as community endoscopists may also be questioned. While the study was undertaken in a nonacademic hospital, it is a tertiary oncology center whose gastroenterologists are expected to have greater-than-average experience in the detection, diagnosis, and resection of colorectal neoplastic lesions. In Por- tugal, most of the screening and diagnostic colonoscopies are performed in private hospitals and clinics, and so the results of this study may lack generalizability to these "true" community colonoscopists.

Nevertheless, this study presents relevant real-life results from a Portuguese tertiary nonacademic hospital, and it is interesting to contrast it with a 2018 Dutch study, which evaluated for the first time the real-time accuracy of diminutive polyps' optical diagnosis using both the NICE and WASP classifications [4]. Despite having the same overall aim, the study settings were very different. Dutch endoscopists were accredited by the Dutch Bowel Cancer Screening Program and underwent intensive image-based and real-time training and testing before inclusion in the study; only $70 \%(n=27)$ of the invited endoscopists completed this testing phase. Over 4,500 diminutive polyps were analyzed, and the threshold NPV for diminutive neoplastic polyps (ADs and SSLs) in the sigmoid and rectum was achieved (90.8\%, 95\% CI 88.692.6). Interestingly, in multivariate analysis, endoscopists who analyzed a greater number of polyps $(>120)$ also had improved accuracy (OR 1.85, 95\% CI 1.25-2.76). This could be a reason for the poorer results by Castela et al. [1], as only 290 polyps were analyzed by 13 endoscopists. As the ongoing application of optical diagnosis in between study periods was not monitored, it is possible that many endoscopists did not progress enough in the learning curve to achieve competency.

Despite their differences, both studies point in the same direction. The WASP classification can diagnose SSLs with excellent accuracy, and going forward, it seems logical to include it in the implementation of the "resectand-discard" and "diagnose-and-leave" policies. As with any technology, training in optical diagnosis is necessary [5]. Even without formal training or feedback, Castela et al. [1] could demonstrate a tendency for improvement of NBI optical diagnosis after 1 year of unmonitored use by endoscopists with no previous NBI experience. Vleugels et al. [4] show that even highly selected and interested endoscopists require auditing and continued practice to ensure that competence is maintained.

The minimum thresholds for the "resect-and-discard" policy can be achieved, at least by some endoscopists [2] Optical diagnosis accuracy is dependent on experience, training, and continued regular use. However, some questions remain: can all endoscopists consistently achieve minimal thresholds after an adequate learning curve? Is monitoring, feedback, and a minimum number of regular examinations necessary to maintain competen$c y$ ? Future investigation must focus on the characteriza- 
tion of the learning curve of this technology, the most efficient mode of training and auditing, and whether appropriate practice can bring any endoscopist to competency.

\section{Disclosure Statement}

The authors have no conflicts of interest to declare.

\section{References}

Optical Diagnosis of Diminutive Colorectal Polyps
1 Castela J, Mão de Ferro S, Rosa I, Lage P, Ferreira S, Pereira Silva J, et al. Real-time optical diagnosis of colorectal polyps in the routine clinical practice using the NICE and WASP classifications in a non-academic setting. GE PortJGastroenterol.DOI:10.1159/000495258.

2 Abu Dayyeh BK, Thosani N, Konda V, Wallace MB, Rex DK, Chauhan SS, et al.; ASGE Technology Committee. ASGE Technology Committee systematic review and meta-analysis assessing the ASGE PIVI thresholds for adopting real-time endoscopic assessment of the histology of diminutive colorectal polyps. Gastrointest Endosc. 2015 Mar;81(3):502.e116.

3 IJspeert JE, Bastiaansen BA, van Leerdam ME, Meijer GA, van Eeden S, Sanduleanu S, et al.; Dutch Workgroup serrAted polypS \& Polyp- osis (WASP). Development and validation of the WASP classification system for optical diagnosis of adenomas, hyperplastic polyps and sessile serrated adenomas/polyps. Gut. 2016 Jun;65(6):963-70.

4 Vleugels JL, Dijkgraaf MG, Hazewinkel Y, Wanders LK, Fockens P, Dekker E, et al.; DISCOUNT study group. Effects of Training and Feedback on Accuracy of Predicting Rectosigmoid Neoplastic Lesions and Selection of Surveillance Intervals by Endoscopists Performing Optical Diagnosis of Diminutive Polyps. Gastroenterology. 2018 May;154(6): 1682-1693.e1.

5 Pimentel-Nunes P, Buxbaum J. Internet based e-learning systems: a tool for the future in endoscopy. Endoscopy. 2017 Oct;49(10): 936-7. 